Research Article

\title{
Enhancement of Fibroblasts Outgrowth onto Polycaprolactone Nanofibrous Grafted by Laminin Protein Using Carbon Dioxide Plasma Treatment
}

\author{
Mohammad Ali Sahebalzamani $^{1}$, Mohammad Taghi Khorasani ${ }^{2}$, Morteza Daliri Joupari ${ }^{3}$ \\ ${ }^{1}$ Department of Biomaterials, Faculty of Biomedical Engineering, Science and Research Branch, Islamic Azad University, Tehran, \\ Iran. \\ ${ }^{2}$ Department of Polymeric Biomaterial, Iran Polymer and Petrochemical Institute, Tehran, Iran. \\ ${ }^{3}$ Department of Animal and Marine Biotechnology, National Institute of Genetic Engineering and Biotechnology, Tehran, Iran. \\ Corresponding author. E-mail: or M.sahebalzamani@srbiau.ac.ir; Tel.: +98-2188698282
}

Received: Jun. 10, 2017; Accepted: Aug. 14, 2017; Published: Sep. 7, 2017.

Citation: Mohammad Ali Sahebalzamani, Mohammad Taghi Khorasani, and Morteza Daliri Joupari, Enhancement of Fibroblasts Outgrowth onto Polycaprolactone Nanofibrous Grafted by Laminin Protein Using Carbon Dioxide Plasma Treatment. Nano Biomed. Eng., 2017 , 9(3): I9I-198. DOI: $10.5101 /$ nbe.v9i3.p191-198.

\begin{abstract}
A common approach in tissue engineering is to mimic the architecture of the natural extracellular matrix (ECM). The ECM plays an important role in regulating cellular behaviors by influencing cells with biochemical signals and topographical cues. Nanofibrous constructs have been used extensively as potential tissue engineering platforms. It is generally hypothesized that a close imitation of the ECM will provide a more conducive environment for cellular functions ranging from adhesion, migration, proliferation to differentiation. In this study, the polycaprolactone (PCL) nanofibers designed were then modified by carbon dioxide plasma and laminin in order to enhance the cell adhesion, spreading and proliferation. The samples were evaluated by attenuated total reflectancefourier transform infrared spectroscopy (ATR-FTIR), scanning electron microscope (SEM), contact angle and finally, cell culture. ATR-FTIR structural analysis showed the presence of functional groups on the nanofibrous surfaces. The SEM images showed the average diameter of nanofibers to be about $100-300 \mathrm{~nm}$ for samples. The $82^{\circ}$ difference was obtained in the contact angle analysis, obtained for the laminin-modified nanofibrous mat against the unmodified nanofibrous mat. Cellular investigation showed better adhesion and cell growth and proliferation of laminin-modified nanofibrous samples than other samples. Therefore, the modification of electrospun scaffolds with bioactive protein is beneficial as this can create an environment that consists of biochemical cues to further promote cell adhesion and proliferation.
\end{abstract}

Keywords: Porous nanofibrous polycaprolactone; Physical adsorption; Carbon dioxide plasma treatment; Enhance the cell adhesion

\section{Introduction}

Tissue engineering is a rapidly growing area that aims to create, repair or replace tissues and organs by using combinations of cells, biomaterials, and biologically active molecules.

The biomaterial scaffold plays a pivotal role in most tissue engineering strategies. To guide the organization, growth and differentiation of cells in tissue engineered constructs, the biomaterial scaffold should be able 
to provide not only a physical support for the cells but also the chemical and biological cues needed in forming functional issues [1]. Polycaprolactone (PCL) is a biomaterial that is used in a variety of applications including surgical sutures, wound dressing, drug delivery and tissue engineering. This is due to its specific properties such as good biocompatibility, biodegradability, non-toxicity as well as appropriate mechanical strength. However, this material is a hydrophobic polyester which should be modified with other materials until it improves its cell adhesion and hydrophilicity properties [2,3]. It has been scientifically proved the extracellular matrix (ECM) mimics improve the attachment, proliferation and the viability of the cultured cells. Electro-spinning has been recently developed into a technique to prepare nanofibers with the diameter ranging from tens of nanometers to several microns [4]. The electro-spun fibrous mats also show extremely high surface area and large porosity. Besides, the fibrous structure of the electro-spun mats mimics the extracellular matrix $[5,6]$.

Some natural materials such as collagen, fibronectin and laminin have been reported as scaffold modifiers. Controlling surface properties is so important for the high performance of adhesion [7]. Biomaterials wettability is a necessary factor in the surface modification of materials. Also, surface modification is an integral element for better growth and proliferation of cells on the nanofiber scaffold. Plasma-surface modification (PSM) is an effective and economical surface treatment technique for many materials and of growing interests in biomedical engineering. Nonthermal and low-pressure plasma have been used in a series of surface treatment applications. The majority of plasma-assisted technologies are based on low pressure processes [8]. The treatment of polymeric materials with plasma is a frequently used technique to accomplish surface modifications that affects chemical composition as well as surface topography [9]. Moreover, microwave discharges are routinely employed in the processing of materials to deposit films as well as coatings [10]. Laminin is a component of the basement membrane and contains the RGD sequence (contains the three-amino-acid sequence arginineglycine-aspartic acid binding sequence that reacts with integrin receptors on the growth cone and promotes cellular adhesion) that aids in cell adhesion and the five aminoacid sequence isoleucine-lysine-valine-alaninevaline sequence that controls neurite outgrowth [11]. Ko et al. used laminin as the contact guidance biochemical cues for axonal outgrowth [12]. Laminin is one of the ECM component that is continuously synthesized after nerve injury and it plays a crucial role in cell migration, differentiation and axonal growth [13]. For example, myelination in the peripheral nervous system (PNS) is affected by laminin. Studies have described that even as Schwann cells could proliferate and migrate along axons, differentiation of myelinating phenotype was not observed without the presence of laminin [14]. Furthermore, in-vitro experiments have shown that neurite outgrowth is enhanced on scaffolds that were covalently bound with laminin [15]. These studies showed that in-vitro directional guidance of the neurite outgrowth was achieved and enhanced using scaffolds that were physically adsorbed with laminin. Improved axonal outgrowth has also been observed in nerve guides filled with laminin gel as well. Therefore, the incorporation of laminin onto nanofibers can potentially improve the rate of nerve regeneration [16-18].

In this research, the modified PCL nanofibers were obtained through the plasma radiation method with carbon dioxide gas. The samples were evaluated by attenuated total reflectance-fourier transform infrared spectroscopy (ATR-FTIR), scanning electron microscope (SEM), water contact angle and also the cell culture with fibroblasts cells.

\section{Materials and Methods Nanofiber preparation}

PCL with $80,000(\mathrm{~g} / \mathrm{mol})$ molecular weight was purchased from Sigma-Aldrich (St. Louis, MO), and hexafluoro-2-propanol (HFP), the solvent system for PCL was purchased from Sigma-Aldrich and used as received without further purification. Electrospinning apparatus used in this study was prepared from Asia nano meghyas company (Iran). PCL was dissolved at determined concentration in HFP. The PCL solution $(9 \% \mathrm{w} / \mathrm{v})$ was contained in a glass syringe controlled by syringe pump. A positive high voltage source through a wire was applied at the tip of a syringe needle. In this situation, a strong electric field was generated between PCL solution and a collector. When the electric field reached a critical value with increasing voltage, mutual charge repulsion overcame the surface tension of the polymer solution and an electrically charged jet was ejected from the tip of a conical shape as the Taylor cone.

The solution was electrospun from a $10 \mathrm{~mL}$ syringe with a needle diameter of $15 \mathrm{~mm}$ and mass flow rate 




Fig. 1 Schematic of modification with laminin by carbon dioxide plasma treatment and physical adsorption.

Table 1 Parameters for nano fibers preparation

\begin{tabular}{cccccc}
\hline $\begin{array}{c}\text { Syringe diameter } \\
(\mathrm{mm})\end{array}$ & $\begin{array}{c}\text { Dram speed } \\
(\mathrm{rpm})\end{array}$ & $\begin{array}{c}\text { Injected speed } \\
(\mathrm{mL} / \mathrm{min})\end{array}$ & $\begin{array}{c}\text { Syringe tip distance } \\
\text { to dram }(\mathrm{mm})\end{array}$ & Voltage $(\mathrm{kV})$ & Temperature $\left({ }^{\circ} \mathrm{C}\right)$ \\
\hline 15 & 1,000 & 1 & 75 & 20 & 25 \\
\hline
\end{tabular}

of $1 \mathrm{~mL} / \mathrm{h}$. A high voltage $(20 \mathrm{kV})$ was applied to the tip of the needle attached to the syringe when a fluid jet was ejected. The linear rate of the rotating disk (drum) was set to $1,000 \mathrm{rpm}$. The resulting fibers were collected on 15-mm cover slips placed on respective collectors. The used parameters for this nanofibers preparation can be seen in Table 1 .

Ultra-fine fibers were formed by narrowing the ejected jet fluid as it underwent increasing surface charge density due to evaporation of the solvent. An electrospun PCL nanofibrous mat was carefully detached from the collector and dried in vacuum for 2 days at room temperature to remove solvent molecules completely.

\section{Electrospun fiber modification}

Vacuum dried PCL fibers were cut to fit in 24well plates; plasma were treated in carbon dioxide and inductively coupled radio frequency (RF) plasma cleaner (DIENER Electronic GmbH, Germany) for 2 min at a RF power of $100 \mathrm{~W}$ to the surface of the fibers. Plasma treated fibers were then incubated in a $10 \mathrm{mg} / \mathrm{mL}$ laminin solution overnight at $4{ }^{\circ} \mathrm{C}$ with gentle shaking for $45 \mathrm{~min}$. Physically adsorbed samples were then immersed in $75 \%$ ethanol overnight, and the samples were kept sterile for cell culture studies or dried completely for characterization.

\section{Fourier transmission infrared spectroscopy (FTIR)}

The samples were examined by FTIR (ATR-FTIR, THERMO NICOLET, NEXUS 670, USA) before and after adjustment. The samples were scratched into powder, produced as pellet using $\mathrm{KBr}$, and then investigated.

\section{Scanning electron microscopy (SEM)}

The surface characteristics of various modified and unmodified films were studied by scanning electron microscopy (SEM; TScan, VEGA, Czech) to analyze the changes in the surface morphology. The films were first coated with a gold layer (Joel fine coat, ion sputter for $5 \mathrm{~min}$ ) to provide surface conduction before scanning. Energy dispersive X-ray analysis was conducted to investigate the existence and distribution of nitrogen in the chemically modified PCL nanofibers so as to confirm the introduction of amino groups and the surface analysis of PCL and laminin-modified PCL nanofibers was carried out. The carbon, hydrogen and nitrogen ratios were determined in different regions of the samples using an EDX detector connected to a scanning electron microscope (SEM) (TScan,VEGA,Czech).

\section{Water Contact angle measurement}

The sample surfaces static contact angles were investigated by a contact-angle-measuring device (OCA 15 plus, Dataphysics) following the sessile drop method.

\section{Cellular analysis}

In this step, the best samples were prepared to evaluate for cell culture assay by fibroblast cells according to the International Organization for Standardization 10993 standards. Tissue culture 
polystyrene (TCPS) were well cleaned and sterilized by the autoclave method. Individual samples were placed in Petri dishes using a sterilized pincer; $3 \mathrm{cc}$ of the cell suspension was removed by pipette and poured into the control and experimental samples. Thereafter, all of the samples were placed separately in an incubator at $37^{\circ} \mathrm{C}$ for 24 and $48 \mathrm{~h}$. For microscopic study, the cultured scaffolds with cells were washed by PBS and then fixed by glutheraldehyde $(2.5 \%)$ at $4{ }^{\circ} \mathrm{C}$ for $2 \mathrm{~h}$. The samples were dehydrated with graded concentration $(50-100 \% \mathrm{v} / \mathrm{v})$ of ethanol and kept in a fume hood for air drying and use for SEM observation. The samples were coated with gold and investigated by a scanning electron microscope (TScan,VEGA,Czech). All data are expressed as mean standard error of the mean unless noted (Student's t-tests were conducted with a significance level of $p<0.05$ ). A minimum of three replicate samples were used for all experiments.

\section{Results and Discussion FTIR results}

The results of the unmodified nanofibrous sample from the ATR-FTIR spectrum, the modified nanofibrous sample with carbon dioxide plasma and the nanofiber sample modified with laminin are shown in Fig. 2 (a)-(c). In Fig. 2(a), the band was in 1,726/cm due to abundant ester $\mathrm{C}=\mathrm{O}$ bonds in the structure of the PCL. The stretching band in $1,240-1,170 / \mathrm{cm}$ was shown to be related to the $-\mathrm{C}-\mathrm{O}-\mathrm{C}-$ group, and the stretching band in 2,800-2,900/cm was shown to be related to the $-\mathrm{CH}_{2}$ groups. Fig. 2(b) illustrates the ATR-FTIR spectra of modified nanofiber carbon dioxide plasma. The spectra shows the strong band in $1,650 / \mathrm{cm}$ was related to the $\mathrm{C}=\mathrm{O}$ group, and that the stretching bands in 2,800-2,900/cm was related to the $-\mathrm{CH}_{2}$ groups. The stretching band in 3,431 / $\mathrm{cm}$ was related to the $\mathrm{OH}$ group. Fig. 2(c) also shows the strong band in $1,722 / \mathrm{cm}$ was related to the $\mathrm{C}=\mathrm{O}$ group, and that the stretching band in $2,800-2,900 / \mathrm{cm}$ was related to the $-\mathrm{CH}_{2}$ groups. The stretching bands in $3,408 / \mathrm{cm}$ and $3,761 / \mathrm{cm}$ were related to the $\mathrm{OH}$ and $\mathrm{NH}$ groups due to the effect of laminin treatment.

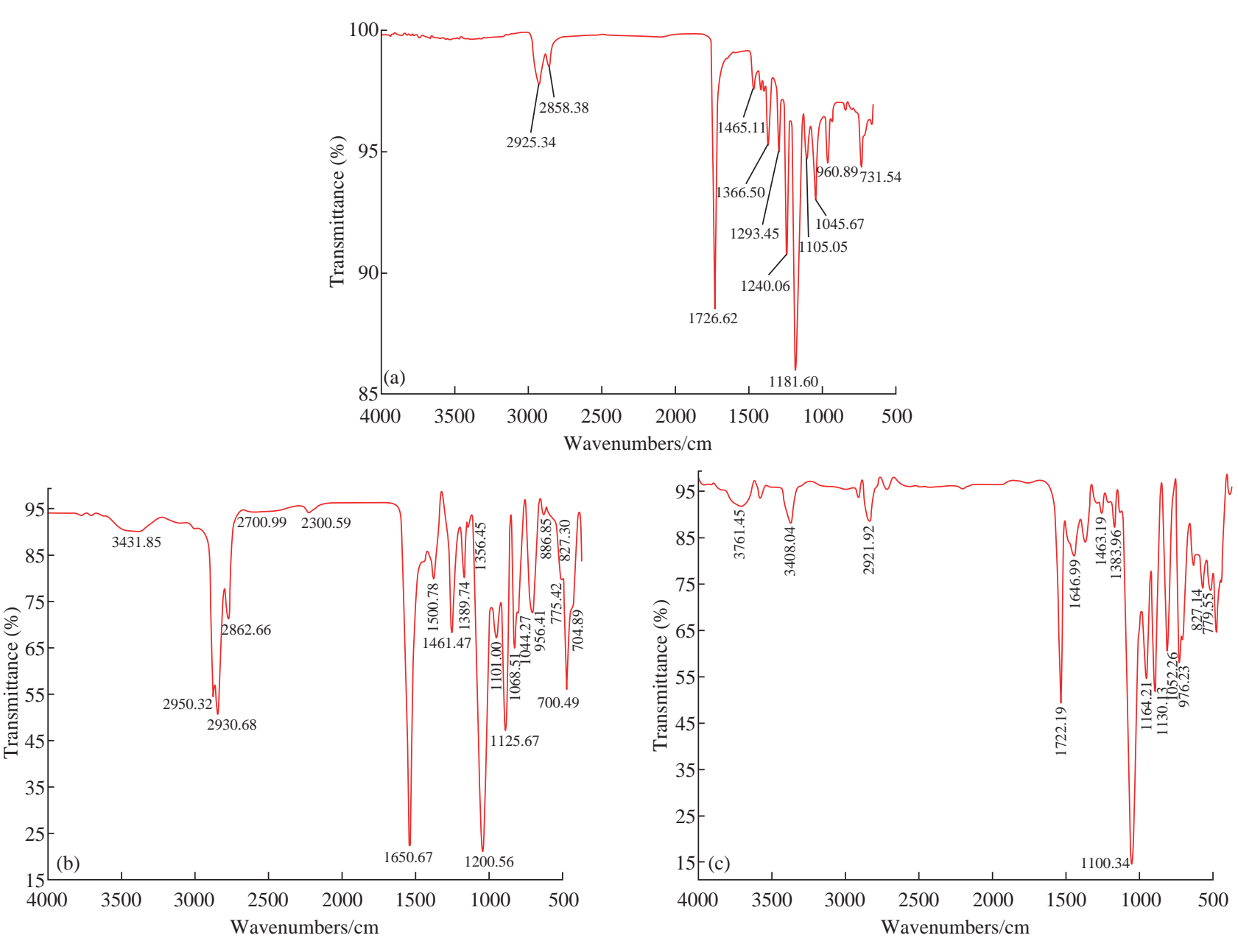

Fig. 2 ATR-FTIR analysis of (a) the unmodified nanofibrous mat; (b) the modified nanofibrous mat with carbon dioxide plasma; and (c) the modified nanofibrous mat with laminin. 



Fig. 3 SEM images of PCL nanofibers. (a) Unmodified nanofibers (Mag: 10,000 ×); (b) Modified by plasma treatment (Mag: 20,000 ×); and (c) Laminin-modified nanofibers (Mag: 10,000 ×).

Table 2 Percentage of carbon, nitrogen and oxygen determined by energy dispersive X-ray analysis (EDX)

\begin{tabular}{cccc}
\hline Samples & Elements weight of carbon (\%) & Elements weight of nitrogen (\%) & Elements weight of oxygen (\%) \\
\hline PCL scaffold & 74.08 & - & 15.36 \\
Laminin modified PCL mat & 67.85 & 5.35 & 17.41 \\
\hline
\end{tabular}

\section{SEM investigations}

Fig. 3 (a)-(c) shows the SEM images of the unmodified and the modified nanofibers by plasma method and modified with laminin. Fig. 3 (a) \& (b) show the non-modified and modified nanofibrous mat prepared by the electrospinning method and modified by plasma method. The smooth and homologous nanofibers are clearly shown. The average size for the unmodified, modified with plasma and the lamininmodified nanofibers (Fig. 3(c)) were about 130, 150 and $190 \mathrm{~nm}$, respectively. Table 2 shows the elemental analysis for unmodified and modified mats. Results demonstrated the presence of $\mathrm{NH}_{2}$ groups on the laminin modified PCL nanofibrous mat. Consequently, these results showed that grafted laminin using carbon dioxide plasma treatment in PCL nanofibrous mat were modified successfully.

\section{Water contact angle results}

Table 3 and Fig. 4 show the contact angle obtained for the unmodified and the modified nanofibers. The contact angles of $112^{\circ}$ and $67^{\circ}$ were obtained for the unmodified and the modified nanofibrous mat with carbon dioxide plasma, respectively. The contact angle of $30^{\circ}$ was obtained for the laminin-modified nanofibrous mat. The $82^{\circ}$ difference in the contact angle, obtained for the laminin-modified nanofibrous mat than the unmodified nanofibrous mat, showed
Table 3 Effect of surface modification on contact angle of nanofibrous samples

\begin{tabular}{cc}
\hline Samples & Contact angle $(\operatorname{deg} \theta)$ \\
\hline PCL nanofibrous mat & 112.1 \\
Plasma modified \\
$\begin{array}{c}\text { PCL nanofibrous mat } \\
\text { Laminin modified } \\
\text { PCL nanofibrous mat }\end{array}$ & 67.5 \\
\hline
\end{tabular}

a better hydrophilicity of the modified mat than the unmodified ones.

\section{Cellular study}

The cellular behavior on a biomaterial is an important factor in determining the biocompatibility. The first physiological process that occurs within the initial stages of exposure is the adsorption of biomolecules onto the surface, and this is usually followed by cellular interactions. The whole process of adhesion and spreading of the cells after contact with biomaterials consists of cell attachment, growth of filopodia, cytoplasmic webbing and flattening of the cell mass, and the ruffling of peripheral cytoplasm, which progress in a sequential fashion $[19,20]$.

Table 4 and Fig. 5 indicate the MTT assay for TCPS (control), the unmodified, the modified by plasma treatment with carbon dioxide and the laminin physically absorbed nanofibrous mat. The 
Table 4 MTT analysis of the samples

\begin{tabular}{lccc}
\hline $\begin{array}{c}\text { Physically adsorbed } \\
\text { laminin nanofibrous mat }\end{array}$ & PCL nanofibrous mat & TCPS (control) & Samples \\
\hline 145 & 112 & 100 & Viability (\%) \\
\hline & (b) & & (c) \\
\hline
\end{tabular}

Fig. 4 (a) Contact angle images of PCL nanofibrous mat; (b) Carbon dioxide plasma modified PCL nanofibrous mat; and (c) Lamininmodified PCL nanofibrous mat.
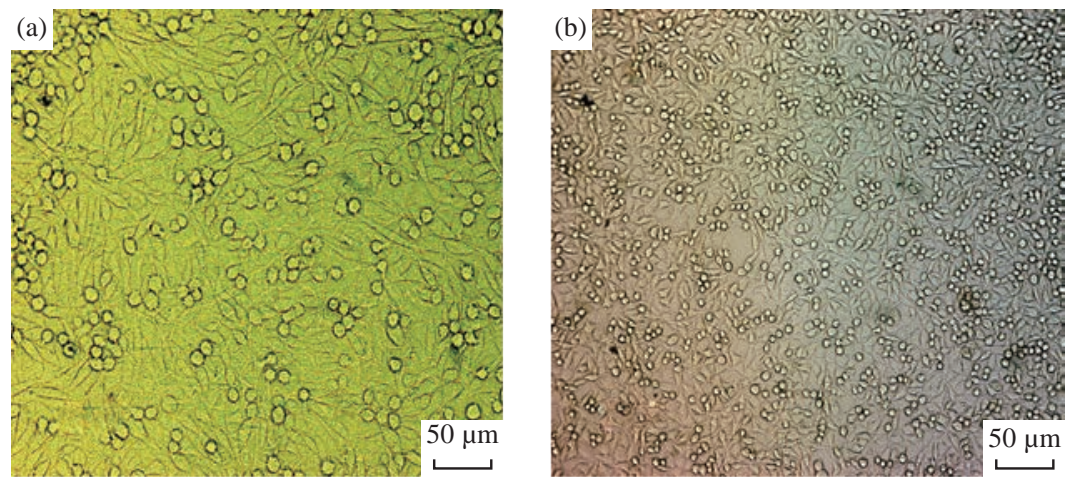

Fig. 5 Fibroblast cells growth on the samples: (a) Control (TCPS); and (b) Coated nanofiber.

results showed a high viability for the samples of the modified by plasma treatment with carbon dioxide and physically adsorbed laminin nanofibrous mat, but the physically adsorbed laminin nanofibrous mat showed a better viability than the other samples.

Fig. 6 shows SEM images of the fibroblasts seeded on the samples. Fig. 6 (a) is related to the unmodified and Fig. 6 (b) is related to the modified by plasma treatment with carbon dioxide. Fig. 6 (c), (d) and (e) are related to the laminin-modified nanofibrous mat at different magnifications. Cellular images showed good growth in the vicinity of modified nanofibers especially the laminin-modified nanofibrous mat. Cell culture assay showed a high attachment for the samples of modified by plasma treatment with carbon dioxide and the laminin-coated nanofibrous mat, but the laminincoated nanofibrous mat showed a better growth than the other samples. Also, these samples caused more cells to proliferate.

In fact, we could see laminin was well characterized to induce cell attachment and outgrowth in this study. Also, we fabricated nano-scaled scaffolds that contained laminin to provide the support and functional biochemical signals for enhancing fibroblasts outgrowth. Laminin was successfully coupled onto PCL nanofibers. Hence, the enhancement of fibroblasts outgrowth using nano-structured scaffolds by laminin was evident.

In a tissue engineering approach, an engineered scaffold loaded with a specific cell type may promote functional restoration. Surface and bulk properties of a well-designed scaffold, similar to the environmental cues in the extracellular matrix, can provide appropriate signals for cell growth, differentiation and subsequent tissue formation. Surface physicochemical properties, such as topography, surface charge and protein adsorption/immobilization/release, have been shown to influence cell behavior. The electrospun nanofibrous scaffolds have gained considerable interest because the architecture is similar to the naturally occurring 

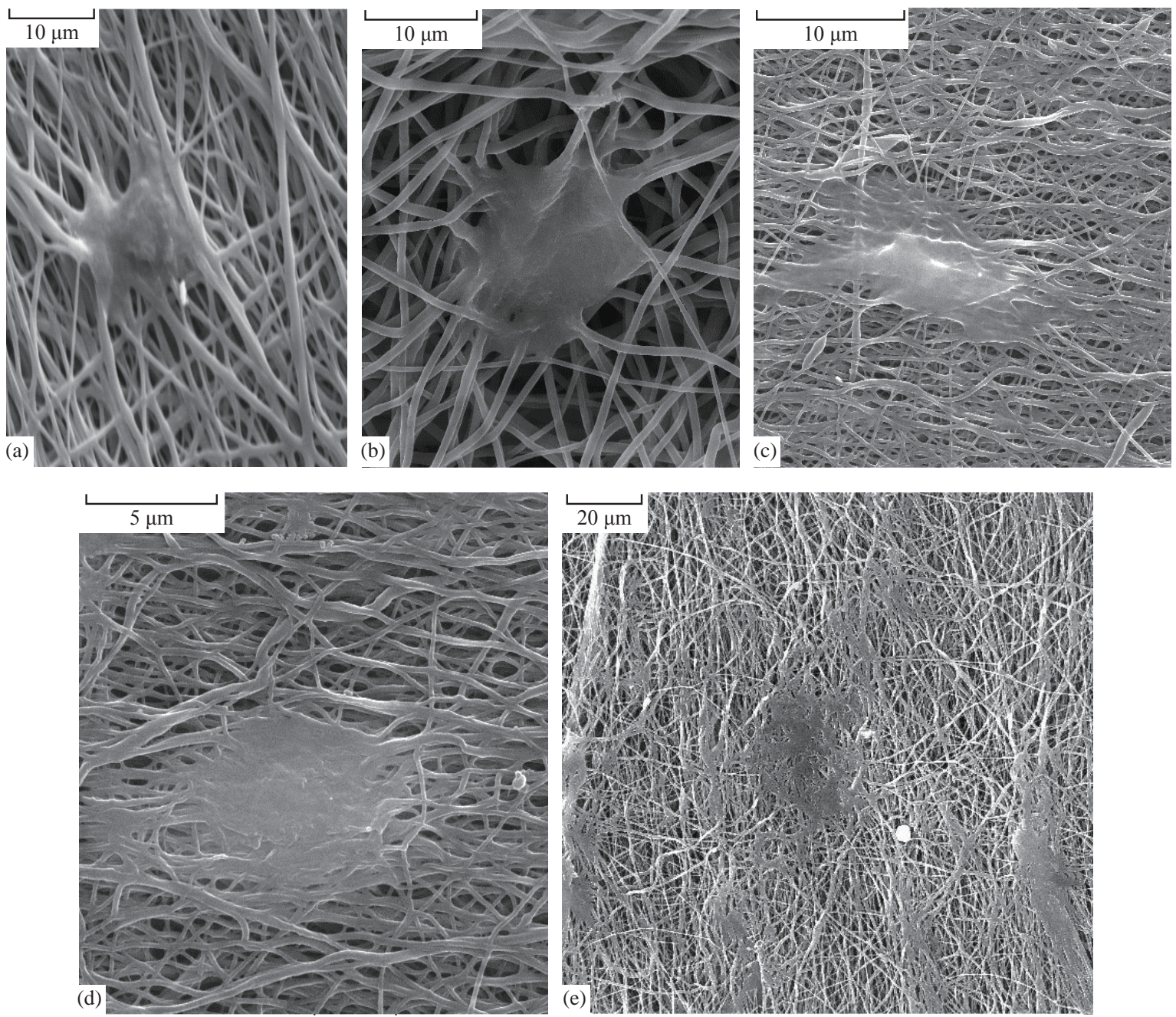

Fig. 6 Fibroblast cells growth on the samples: (a) Unmodified $(5,000 \times)$; (b) modified by plasma treatment with carbon dioxide $(3,000$ $\times$ ); (c)-(e) laminin-modified nanofibrous mat in different magnifications ((c): 3,000 ×; (d): 5,000 ×; (e):10,000×).

protein fibrils in the extracellular environment [21-23]. Each individual nano-scale fiber has a high surface to volume and aspect ratio allowing more surface area contact of the scaffold with the cell. The physical and biological properties of the scaffold are dependent on the material used for electrospinning and its properties such as surface wettability, mechanical properties and degradation [22-25]. The properties of the scaffolds can be manipulated by copolymerization or polymer blending of various biodegradable, non-biodegradable, synthetic and/or natural materials [25-27].

In fact, surface modification is necessary for better growth and proliferation of cells on the nanofiber scaffold. In this research, we will focus on the increase in surface hydrophilicity in two steps, first with plasma and then with coating.

\section{Conclusions}

PCL nanofibers with an average size of about
$200 \mathrm{~nm}$ were designed and successfully modified by the plasma treatment method with the carbon dioxide gas. The plasma-modified nanofibrous mats subsequently coated with ECM protein to improve biocompatibility. ECM protein, namely laminin, was covalently bound to PCL nanofibers and compared for its efficacy in enhancing cellular outgrowth. An $81^{\circ}$ difference in the contact angle, obtained for the modified samples, showed a better hydrophilicity of the modified nanofibers than the unmodified ones. Cellular investigations showed better adhesion, growth and viability in the laminin-modified nanofibrous mat than the PCL nanofiber sample (unmodified) and PCL modified by plasma treatment with carbon dioxide sample. These modified nanofibrous mat could well be used for tissue engineering. This paper demonstrates that scaffolds could be fabricated by electrospinning to produce nano-scale architectured scaffolds and could be easily functionalized with biochemical cues (e.g., laminin) to further enhance fibroblasts outgrowth for 
potential applications in tissue repair. Importantly, modified electrospun nanofibers as compared to normal or unmodified sample were a useful and easy method to modify biomaterials to produce biomimetic scaffolds that had both topographical and biochemical cues. In the analysis, the smooth and homology modified nanofibers were clearly shown in the figures. Cellular images showed well growth in the vicinity of nanofibers, especially the coated nanofibers. These laminin coated nanofibers could be used well for tissue engineering.

\section{Conflict of Interests}

The authors declare that no competing interest exists.

\section{References}

[1] R. Langer, D.A Tirrell, Designing materials for biology and medicine. Nature, 2004, 428: 487-492.

[2] M.S. Austero, A.E. Donius, U.G.K. Wegs, et al., New crosslinkers for electrospun chitosan fibre mats. I. Chemical analysis. J R Soc Interface, 2012, 9(75): 25512562.

[3] L.A. Smith, P.X. Ma, Nano-fibrous scaffolds for tissue engineering. Colloids Surf, Biointerfaces, 2004, 39: 125130.

[4] W. Wang, S. Itoh, K. Konno, et al., Effects of Schwann cell alignment along the oriented electrospun chitosan nanofibers on nerve regeneration. Biomed Mater Res A, 2011, 91: 994-1005.

[5] A. Ndreu, L. Nikkola, H. Ylikauppila, et al., Electrospun biodegradable nanofibrous mats for tissue engineering. Nanomedicine (Lond), 2008, 3(1): 45-60.

[6] H. Cao, T. Liu, and Y.C. Sing, The application of nanofibrous scaffolds in neural tissue engineering. Advanced Drug Delivery Reviews, 2009, 61: 1055-1064.

[7] E. Biazar, S. Heidari, M.A. Sahebalzamani, et al., Design of oriented porous PHBV scaffold as a neural guide. International Journal of Polymeric Materials and Polymeric Biomaterials, Taylor \& Francis Group, 2014, 63: 753-757.

[8] B.J. Pfister, T. Gordon, Biomedical engineering strategies for peripheral nerve repair: Surgical applications, state of the art, and future challenges. Critical Reviews ${ }^{\mathrm{TM}}$ in Biomedical Engineering, 2011, 39(2): 81-124.

[9] S. Heidari, E .Biazar, M.A. Sahebalzamani, et al. The healing effect of unrestricted somatic stem cells loaded in nanofibrous PHBV scaffold on full-thickness skin defects. Journal of Biomaterials and Tissue Engineering (JBT), 2014, 4(1): 20-27.

[10] M. Santosh, D. Kumar Bishi, et al., Nanofibers coated on acellular tissue engineered bovine pericardium supports differentiation of mesenchymal stem cells into endothelial cells for tissue engineering. J Nanomedicine, 2014, 9(5): 623-634.

[11] R. Jayakumar, M. Prabaharan, S. Nair, et al., Novel chitin and chitosan nanofibers in biomedical applications. Biotechnol Adv, 2010, 28(1): 142-150.
[12] J. Ko, H. Yin, J. An, et al., Characterization of crosslinked gelatin nanofibers through electrospinning. Macromol Res, 2010, 18(2): 137-143.

[13] M.A. Sahebalzamani, E. Biazar, M. Shahrezaie, et al., Surface modification of PHBV nanofibrous mat by Laminin protein and its cellular study. International Journal of Polymeric Materials and Polymeric Biomaterials, 2015, 64(3): 149-154.

[14] M. Mekhail, K. Wong, D.T. Padavan, et al., Genipincross-linked electrospun collagen fibers. J. Biomater Sci Polym Ed, 2011, 22(17): 2241-2259.

[15] M.E. Frohbergh, A. Katsman, G.P. Botta, et al., Electrospun hydroxyapatite-containing chitosan nanofibers crosslinked with genipin for bone tissue engineering. Biomaterials, 2012, 33(36): 9167-9178.

[16] M. Pakravan, M.C. Heuzey, A. Ajji, A fundamental study of chitosan/PEO electrospinning. Polymer, 2011, 52(21): 4813-4824.

[17] Z.G. Chen, P.W. Wang, B. Wei, et al., Electrospun collagen-chitosan nanofiber: A biomimetic extracellular matrix for endothelial cell and smooth muscle cell. Acta Biomater. 2010, 6(2): 372-382.

[18] S. Heidari, Effects of chitosan cross linked nano-fibrous PHBV scaffold combined with mesenchymal stem cells on healing of full-thickness skin defects. J biomed nanotechnol, 2013, 9:1471-1482.

[19] M. Dadsetan, H. Mirzadeh, N. Sharifi Sanjani, et al., Cell behavior on laser surface-modified PET in vitro. $J$. Biomed. Mater. Res., 2001, 57: 183-189.

[20] R. Mehdinavaz, S. Shakhesi, S. Najarian, et al., Preparation of mineralized electrospun fibers as a biomimetic nanocomposite. Int. J. Polym. Mater. Polym. Biomater, 2014, 63: 576-585.

[21] D.N. Deal, J.W. Griffin, M.V. Hogan, Nerve conduits for nerve repair or reconstruction. J Am Acad Orthop Surg., 2012, 20: 63-68.

[22] M.T. Khorasani, H. Mirzadeh, S. Irani, Comparison of fibroblast and nerve cells response on plasma treated poly (L-lactide) surface. Journal of Applied Polymer Science, 2009, 112: 3429-3435.

[23] Z. Xiaoduo, G. Mingyong, et al., Nanofiber scaffolds facilitate functional regeneration of peripheral nerve injury. Nanomedicine: Nanotechnology, Biology, and Medicine, 2013, 9: 305-315.

[24] Y. Lee, T. Livingston Arinzeh, Electrospun nanofibrous materials for neural tissue engineering, review. Polymers, 2011, 3: 413-426.

[25] M. Montazeri, N. Rashidi, E. Biazar, et al., Compatibility of cardiac muscle cells on coated-gelatin electrospun polyhydroxybutyrate/valerate nano fibrous film. Bioscience, Biotechnology Research ASIA, 2011, 8(2): 515-521.

[26] F. Chen, P. Huang, and X.-M. Mo, Electrospinning of heparin encapsulated P(LLA-CL) core/shell nanofibers. Nano Biomed. Eng., 2010, 2: 56-60.

[27] C. He, L. Zhang, H. Wang, et al., Physical-chemical properties and in vitro biocompatibility assessment of spider silk, collagen and polyurethane nanofiber scaffolds for vascular tissue engineering. Nano Biomed. Eng., 2009, $1,80-88$.

Copyright $(2017$ Mohammad Ali Sahebalzamani, Mohammad Taghi Khorasani, and Morteza Daliri Joupari. This is an openaccess article distributed under the terms of the Creative Commons Attribution License, which permits unrestricted use, distribution, and reproduction in any medium, provided the original author and source are credited. 